\title{
Quality Control of Withania somnifera and its Marketed Formulations by Validation through High Performance Thin Layer Chromatography
}

Sonia Hooda ${ }^{1}$, Shweta Parihar ${ }^{1}$, Saloni Kakkar ${ }^{2}$, Meenu Bhan ${ }^{2 *}$

${ }^{1}$ Research Scholar, Department of Pharmaceutical Sciences, Maharshi Dayanand University, Rohtak, Haryana, India

${ }^{2}$ Assistant Professor, Department of Pharmaceutical Sciences, Maharshi Dayanand University, Rohtak, Haryana, India

DOI: $10.36347 /$ sajp.2022.v11i01.004

| Received: 09.12.2021 | Accepted: 13.01.2022 | Published: 16.01.2022

*Corresponding author: Meenu Bhan

Assistant Professor, Department of Pharmaceutical Sciences, Maharshi Dayanand University, Rohtak, Haryana, India

\section{Abstract}

Withania somnifera is the most valuable herbaceous plant, also known as Ashwagandha used in the traditional systems of Indian medicine having many therapeutic effects. It is obtained from the dried roots and stems of Withania somnifera belonging to family Solanaceae. The active chemical constituents of Withania somnifera are withaferin A, withanolide A, withasomniferin-A, isopelletierine, withasomidienone, tropine, withanone, cuscohygrine, anaferine, hygrine, anahygrine, somniferine, mesoanaferine, etc. It is used as a liver tonic, aphrodisiac, in asthma, emaciation, in bronchitis and ulcers. It has life prolonging, rejuvenating effect \& also used for the treatment of insomnia, anxiety, skin disease, nervous exhaustion, impotency, enhancing memory and insulin secretion etc. Marketed formulations of Ashwagandha are Ashwagandharista, Himalaya Ashwagandha, Stresswin, Stresscom, Inlife Ashwagandha capsules, Himalaya massage oil, Ancient Apothecary, KSM 66 Ashwagandha, Vigomax, Baidyanath Ashwagandha Amrita 450 $\mathrm{ml}$, Vital plus, Amrutha kasthuri and Brento etc. To maintain the quality of marketed formulations validation should be done. Different validation parameters such as LOQ, LOD, range \& linearity, accuracy, ruggedness and specificity have been studied. Ashwagandha is in demand as a good health promoter is expanding in global market. Many efforts were undertaken to develop a better Withania somnifera variety with a specific chemotype. HPTLC (High performance thin layer chromatography) is most important in evaluating the quantity and quality of herbal drug. This technique is modern \& effective form of TLC which is used for qualitative and quantitative analytical determination of analytes. It is the simple, rapid, precise, specific, robust and accurate technique. The effect of different extraction methods on marker compound Withaferin-A \& other than Withaferin-A, effect of different climatic zones on phytochemical profile of Withania somnifera, different mobile phase used and quantification \& validation of withaferin-A by using HPTLC have also been studied.

Keywords: Ashwagandha, Withania somnifera, marketed formulations, HPTLC, Validation, Withaferin A, Withanolide A.

Copyright ( $\odot 2022$ The Author(s): This is an open-access article distributed under the terms of the Creative Commons Attribution 4.0 International License (CC BY-NC 4.0) which permits unrestricted use, distribution, and reproduction in any medium for non-commercial use provided the original author and source are credited.

\section{INTRODUCTION}

In the present era, mind of people has been changed from synthetic to herbal medicines. With increasing the need of herbal products it is necessary to maintain the quality of them for betterment of human being (Sharma et al., 2008). Various quality control tools are there which are used to ensure the quality of herbal drugs. Both quantitative \& qualitative parameters are required for the quality assurance of them. Techniques such as IR, UV which are commonly used for qualitative determinations whereas HPLC( high pressure liquid chromatography), HPTLC (high performance thin layer chromatography), SFC (supercritical fluid chromatography), GC-MS (gas chromatography-mass spectroscopy), ICP-MS (inductively coupled plasma-mass spectroscopy), thermal analysis, GC-MS (gas chromatography-mass spectroscopy) are used to quantification of herbal medicines for the purpose of quality control (Balekundri et al., 2020).

Withania somnifera also known as Ashwagandha is most valuable herbaceous plant in the traditional systems of Indian medicine having many therapeutic effects (Gupta et al., 2007). It is also called "Indian Winter cherry" or "Indian Ginseng" (Singh et al., 2011). It is a desert plant which grew up in dried and rain-forest regions (Meher et al., 2016). The active chemical constituents of Withania somnifera are withaferin A, withanolide A, ashwagandhine, 
withasomniferin-A, isopelletierine, withasomidienone, tropine, withanone, cuscohygrine, $\beta$-sitosterol D glucoside, gallic acid, anaferine, hygrine, anahygrine, somniferine, rutin, mesoanaferine, etc (Saleem et al., 2020). It has life prolonging, rejuvenating effect $\&$ also used for the treatment of insomnia, anxiety, convulsion, skin disease, inflammatory conditions, nervous exhaustion, impotency, enhancing memory or cognitive and enhance insulin secretion and has been shown to be a successful treatment for cancer cells (Krutika et al., 2016). Some marketed formulations of Withania somnifera are Ashwagandharista, Himalaya ashwagandha, Stresswin, Stresscom, MuscleBlaze Ashwagandha 1000mg Tablet, Inlife Ashwagandha Capsules, Himalaya massage oil, Ancient Apothecary, KSM 66 Ashwagandha, Vigomax, Baidyanath Ashwagandha Amrita $450 \mathrm{ml}$, Vital plus, Amrutha kasthuri and Brento etc. To know the quality of marketed formulations, validation is done. And in this review different extraction methods, solvents used for exytraction, different validation methods and effect of different climatic zones on phytochemical profile on are discussed.

HPTLC is a prominent analytical method for quantification and fingerprinting of marker compounds in herbal medicines because of its simplicity, accuracy, sensitivity and applicability for high throughput screening of herbal medicine. The mobile phase of HPTLC has a high speed capillary flow range (Modi et al., 2016). This technique is superior to more analytical techniques in case of cost and time for analysis (Sonia et al., 2017).

Validation is the technique used for confirming that the scientific technique hired for a particular test is appropriate for its intended use. It is an aspect of quality assurance since it entails a systematic examination of process, facilities and system to see if they execute their intended functions consistently and effectively. For compound evaluation, there are eight parameters which are Robustness, LOD, LOQ, Range \& Linearity, Accuracy, Ruggedness, Specificity and Precision (Ahir et al., 2014).

Robustness is the method which is used to estimate of its volume persist unaltered at little yet intentional convert in technical variables and lay out a sign of its reliability throughout common use (Patil et al., 2017).

Limit of detection defined as the lowest concentration of a specimen in sample which can be identified, still not definitely quantified.

Limit of quantification explained as the smallest concentration of an analyte in a sample with acceptable precision and accuracy can be quantified (Rashmin et al., 2012).

Linearity is defined as its ability to obtain test results which are shortly, or beyond means of wellstated mathematical variation, proportional to the mass of specimen in samples in a given value.

Accuracy of a systematic technique is the extent to which true values agree after providing test results by the method. For estimation of accuracy true value can be obtained in different ways. It expresses the correctness of the method (Patil et al., 2017).

Ruggedness define the degree of reproducibility of test outcome prevail by analyzing the same sample under different test conditions.

Specificity defines the ability of the method to compute accurately and specifically the substance of interest in the sample as impurities (Patil et al., 2017).

Precision refers to the accordance between the single test products when a technique is appeal frequently to the same sample. It is usually indicated as relative standard deviation (Rashmin et al., 2012).

The major component present in Withania somnifera is withaferin-A and minor components are withanolide A and 12 deoxy withastramonolide. This extraction methods and extracting solvents plays an important role in extracting the analytes from the drug.

\section{Effect of different extraction methods on marker compound Withaferin-A \& other than Withaferin-A}

The most commonly used conventional and novel extraction methods for extracting the withaferinA from Withania somnifera with percentage yield is mentioned in table 1and other than withaferin-An are mentioned in table 2. Soxhaltion is based on cell permeation and the extracting solvent is used to solubilize the active ingredients. In maceration technique, coarsely powdered drugs placed in a container \& the menstruum is used for the extraction. These traditional procedures are solvent and time consuming, as well as thermally hazardous. Microwave assisted extraction has been developed and refined for quick extraction for withanolides in response to the increasing demand for more environmental friendly approaches. MAE involves using microwave energy to heat the solvent in contact with the sample. Different extraction techniques \& different solvents show variations in extraction yield due to the polarity of the solvents. By changing the solvent methanol to water in maceration technique, gives high percentage yield of withaferin-A but this technique is very time consuming. 
Table-1: Effect of different extraction methods on marker compound Withaferin-A (Jyothi et al., 2010 and Jain et al., 2010)

\begin{tabular}{|l|l|l|l|l|}
\hline Extraction methods & $\begin{array}{l}\text { Extracting } \\
\text { solvent }\end{array}$ & $\begin{array}{l}\text { Solvent used } \\
(\mathbf{m l} / \mathbf{g})\end{array}$ & $\begin{array}{l}\text { Extraction } \\
\text { time }\end{array}$ & $\begin{array}{l}\text { \%age Yield of } \\
\text { withaferin-A }\end{array}$ \\
\hline Soxhlation & Methanol & 50 & $14 \mathrm{~h}$ & 0.16 \\
\hline Maceration & Water & 50 & $10 \mathrm{~h}$ & 4.80 \\
\hline $\begin{array}{l}\text { Microwave Assisted } \\
\text { Extraction }\end{array}$ & Methanol & 20 & $2 \mathrm{~min}$ & 0.69 \\
\hline
\end{tabular}

Table-2: Effect of different extraction methods on marker compound other than Withaferin-A (Dhanani et al., 2017)

\begin{tabular}{|l|l|l|l|l|}
\hline $\begin{array}{l}\text { Extraction } \\
\text { method }\end{array}$ & $\begin{array}{l}\text { Solvent used for } \\
\text { extraction }\end{array}$ & \%age Yield & $\begin{array}{l}\text { Withanolide A } \\
(\boldsymbol{\mu g} / \mathbf{m g})\end{array}$ & $\begin{array}{l}\mathbf{1 2} \text { deoxy withastramonolide } \\
(\boldsymbol{\mu g} / \mathbf{m g})\end{array}$ \\
\hline \multirow{4}{*}{ Soxhlet } & Water & 9.51 & 1.14 & 0.36 \\
\cline { 2 - 5 } & Ethanol & 9.08 & 3.57 & 1.22 \\
\cline { 2 - 5 } & Water:Ethanol & 9.43 & 1.25 & 0.40 \\
\hline \multirow{2}{*}{$\begin{array}{l}\text { Microwave assisted } \\
\text { extraction }\end{array}$} & Water & 11.18 & 0.59 & 0.20 \\
\cline { 2 - 5 } & Ethanol & 10.01 & 4.35 & 1.39 \\
\cline { 2 - 5 } & Water:Ethanol & 11.39 & 1.09 & 0.28 \\
\hline \multirow{2}{*}{$\begin{array}{l}\text { Ultrasonic } \\
\text { extraction method }\end{array}$} & Water & 9.90 & 0.91 & 0.26 \\
\cline { 2 - 5 } & Ethanol & 2.85 & 6.04 & 2.04 \\
\cline { 2 - 5 } & Water:Ethanol & 9.74 & 1.21 & 0.41 \\
\hline
\end{tabular}

3. Effect of different climatic zones on phytochemical profile of withania somnifera

Different agro-climatic conditions have a significant impact on phytochemical composition in plants. It has been shown that plant physical and chemical behaviour varies greatly depending on the climatic conditions, resulting in differential variance in the active ingredients present in that particular plant mentioned in table 3. Maximum amount of withaferin-A $1.19 \%$ was found in desert region \& withanolide-A content $3.22 \%$ also from desert region. It was observed that when the samples collected from the desert region it has a good phytochemical profile than the other samples collected from the different regions.

Table-3: Effect of different climatic zones on phytochemical profile of Withania somnifera (Kherde et al., 2020)

\begin{tabular}{|l|l|l|l|l|}
\hline Quantification by HPTLC & Coastal Region & Desert Region & Plateau Region & Plains Region \\
\hline Withaferine-A & $0.82 \%$ & $1.19 \%$ & $0.49 \%$ & $0.42 \%$ \\
\hline Withanoloide-A & $2.40 \%$ & $3.22 \%$ & $0.63 \%$ & $1.41 \%$ \\
\hline
\end{tabular}

4. Effect of different solvents of increasing polarity on the extraction of total withanolides (wda, wa, 12wd) from withania somnifera

Different solvents according to their polarity are used. Hexane, chloroform, ethyl acetate and methanol are used to demonstrate the extraction effectiveness of aqueous alcoholic solvents with percentage yield are mentioned in table 4. Highest concentration of three withanolides was yielded by organic solvents chloroform and ethyl acetate. Upscaling process development in the preparation of enriched extracts from Withania somnifera, bioprospecting investigations, crop improvement and quality control could all benefit from an optimised extraction process. Maximum yield was obtained from methanol and minimum from hexane. It was concluded that higher composition of polar solvent gives better extraction yield.

Table-4: Different solvents of increasing polarity on the extraction of total withanolides (Kumar et al., 2010)

\begin{tabular}{|l|l|l|l|l|l|}
\hline Solvent & $\begin{array}{l}\text { \%age yield } \\
\text { of extract }\end{array}$ & $\begin{array}{l}\text { \%age yield of } \\
\text { Withanolide- A }\end{array}$ & $\begin{array}{l}\text { \%age yield of } \\
\text { withaferin-A }\end{array}$ & $\begin{array}{l}\text { \%age yield of } \\
\text { Withanolide- D }\end{array}$ & $\begin{array}{l}\text { Withanolide, } \\
\text { \% in extract }\end{array}$ \\
\hline $\mathrm{Hexane}$ & 1.60 & 0.7184 & NQ* & 0.0060 & 0.7244 \\
\hline $\mathrm{CHCl}_{3}$ & 2.90 & 0.9268 & 0.2705 & 0.1615 & 1.3588 \\
\hline $\mathrm{EtOAc}$ & 6.00 & 1.0182 & 0.1676 & 0.1468 & 1.3326 \\
\hline $\mathrm{MeOH}$ & 12.47 & 0.1762 & 0.0344 & 0.0305 & 0.2410 \\
\hline
\end{tabular}

*Not quantifiable

The mobile phase for quantification of withanolides is chosen depending on the adsorbent material employed as the stationary phase, as well as the physical and chemical properties of the analyte. List of different mobile phases at specific composition for the separation of withanolides are mentioned in table 5 . 
Table-5: List of different mobile phase used for quantification of different withanolides by HPTLC (Srivastava et al., 2008, Sharma et al., 2007, Devkar et al., 2012, Sangwan et al., 2004)

\begin{tabular}{|l|l|l|}
\hline Sr. no. & Mobile phase & Withanolides \\
\hline 1 & Dichloromethane-methanol-acetone-diethyl ether(15:1:1:1v/v) & $\begin{array}{l}\text { Withanolide A, withaferin-A \& } \\
\text { withastraminolide }\end{array}$ \\
\hline 2 & Ethyl acetate-toluene-formic acid(5:5:1)v/v deoxy- & Withanolide A \& withaferin A \\
\hline 3 & $\begin{array}{l}\text { Dichloromethane-toluene-methanol-diethyl ether- } \\
\text { acetone(7.5:7.5:3:1:1) }\end{array}$ & $\begin{array}{l}1,2 \text { deoxy-withastraminolide, withanolide A, } \\
\text { withanolide B \& withaferin-A }\end{array}$ \\
\hline 4 & Chloroform -benzene- methanol-ethyl acetate(74:24::4) & Withanolide A, withaferine A \& Withanone \\
\hline
\end{tabular}

The amount of the marker compound withaferin-A was varying with different plant parts of Withania somnifera. The $\mathrm{R}_{\mathrm{f}}$ values, LOD and LOQ were different in different plant parts of Withania somnifera. Different mobile phases has been used for the quantification of marker compound withaferin-A. Validation and quantification of withaferin-A from different plant parts of Withania somnifera and its marketed formulations by HPTLC are mentioned in table 6 and 7 .

Table-6: Validation and quantification of withaferin-a from different plant parts of W. somnifera by HPTLC (Devkar et al., 2012, Sharma et al., 2007, Srivastava et al., 2008, Mirjali et al., 2009, Nayak et al., 2009, Tomar et al., 2019)

\begin{tabular}{|l|l|l|l|l|l|l|l|l|}
\hline $\begin{array}{l}\text { Plant part } \\
\text { used }\end{array}$ & Mobile phase & $\begin{array}{l}\text { \%age yield of } \\
\text { Withaferin-A }\end{array}$ & $\begin{array}{l}\text { LOD } \\
(\mathbf{n g})\end{array}$ & $\begin{array}{l}\text { LOQ } \\
(\mathbf{n g})\end{array}$ & $\begin{array}{l}\text { Average } \\
\text { recovery } \\
(\%)\end{array}$ & $\begin{array}{l}\text { Precision } \\
(\%) \text { RSD }\end{array}$ & $\begin{array}{l}\text { Absorption } \\
\text { reflection } \\
\text { mode(nm) }\end{array}$ & $\begin{array}{l}\mathbf{R}_{\mathbf{f}} \\
\text { values }\end{array}$ \\
\hline Root & $\begin{array}{l}\text { Dichloromethane-toluene- } \\
\text { methanol-acetone-diethyl } \\
\text { ether (7.5:7.5:3:1:1 v/v) }\end{array}$ & 7.46 & 120 & 350 & 98 & 1.16 & 235 & 0.58 \\
\hline $\begin{array}{l}\text { Whole } \\
\text { plant }\end{array}$ & $\begin{array}{l}\text { Toluene: } \\
\text { ethyl acetate:formic acid } \\
\text { (5:5:1) }\end{array}$ & 1.46 & 120 & 800 & 96.3 & 1.28 & 530 & 0.33 \\
\hline $\begin{array}{l}\text { Plant } \\
\text { tissues }\end{array}$ & $\begin{array}{l}\text { dichloromethane- } \\
\text { methanol- } \\
\text { acetone-diethyl ether }(15+ \\
1+1+1, \text { v/v/v/v) }\end{array}$ & 2.19 & 19.62 & 65.39 & 98.44 & 0.18 & 230 & $0.61 \pm$ \\
\hline $\begin{array}{l}\text { Aerial part } \\
\text { \& Root }\end{array}$ & $\begin{array}{l}\text { Ethyl } \\
\text { acetate/toluene/formic } \\
\text { acid/2-propanol } \\
\text { (7:2:0.5:0.5) }\end{array}$ & 2.2 & 18.28 & 60.31 & 98 & 0.99 & 215 & 0.04 \\
\hline Root & $\begin{array}{l}\text { Toluene-ethyl acetate- } \\
\text { formic acid 5:5:1 }\end{array}$ & 0.0376 & 100 & 800 & 101 & 0.81 & 200 & 0.29 \\
\hline $\begin{array}{l}\text { Root, stem, } \\
\text { leaves }\end{array}$ & $\begin{array}{l}\text { Toluene, ethyl acetate and } \\
\text { acetic acid (60:40:4) }\end{array}$ & 0.99 & 200 & 666 & $203 \pm 1.4$ & 0.69 & 221 & 0.57 \\
\hline
\end{tabular}

Table-7: Validation and quantification of withaferin-a from marketed formulations of $W$. somnifera by HPTLC (Tatke et al., 2010, Bhondave et al., 2014, Mistry et al.,2015, Trivedi et al.,2009)

\begin{tabular}{|c|c|c|c|c|c|c|c|c|}
\hline $\begin{array}{l}\text { Marketed } \\
\text { formulations }\end{array}$ & Mobile phase- & $\begin{array}{l}\text { \%age } \\
\text { yield of } \\
\text { Withafe } \\
\text { rin-A } \\
\end{array}$ & $\begin{array}{l}\text { LOD } \\
\text { (ng) }\end{array}$ & $\begin{array}{l}\text { LOQ } \\
\text { (ng) }\end{array}$ & $\begin{array}{l}\text { Average } \\
\text { recovery } \\
(\%)\end{array}$ & $\begin{array}{l}\text { Precision } \\
(\%)\end{array}$ & $\begin{array}{l}\text { Absorption } \\
\text { reflection } \\
\text { mode }\end{array}$ & $\begin{array}{l}\mathbf{R}_{\mathbf{f}} \\
\text { values }\end{array}$ \\
\hline Churna & Methanol: Chloroform 2:8 & 0.458 & 100 & 300 & $101.98 \pm 0.11$ & 0.006 & $207 \mathrm{~nm}$ & 0.59 \\
\hline Arishta & Methanol: Chloroform 2:8 & 0.137 & 100 & 300 & $103.37 \pm 0.16$ & 0.057 & $207 \mathrm{~nm}$ & 0.59 \\
\hline Capsule & Methanol: Chloroform 2:8 & 0.437 & 100 & 300 & $102.44 \pm 0.12$ & 0.059 & $207 \mathrm{~nm}$ & 0.59 \\
\hline Vati & Methanol: Chloroform 2:8 & 0.698 & 100 & 300 & $103.21 \pm 0.28$ & 0.046 & $207 \mathrm{~nm}$ & 0.59 \\
\hline $\begin{array}{l}\text { Ashvagandhar } \\
\text { ishta }\end{array}$ & $\begin{array}{l}\text { toluene: ethyl acetate: } \\
\text { formic acid: } \\
\text { methanol(6:3:0.1:0.6, } \\
\mathrm{v} / \mathrm{v} / \mathrm{v} / \mathrm{v})\end{array}$ & $\begin{array}{l}81.14 / \\
\pm 1.37 \mathrm{ng} \\
\text { /band }\end{array}$ & $\begin{array}{l}12.04 \\
87\end{array}$ & $\begin{array}{l}36.51 \\
14\end{array}$ & $\begin{array}{l}101.42 / \\
\pm 1.68\end{array}$ & $\begin{array}{l}0.80- \\
0.89\end{array}$ & $474 \mathrm{~nm}$ & $\begin{array}{l}0.37 \pm 0 \\
.02\end{array}$ \\
\hline $\begin{array}{l}\text { Union Total } \\
\text { capsule }\end{array}$ & $\begin{array}{l}\text { chloroform: methanol: } \\
\text { toluene: formic acid }(6.5 \text { : } \\
0.5: 3: 0.25 \mathrm{v} / \mathrm{v} / \mathrm{v} / \mathrm{v})\end{array}$ & 0.722 & $\begin{array}{l}23.81 \\
8\end{array}$ & $\begin{array}{l}72.17 \\
5\end{array}$ & $\begin{array}{l}100.06 \% \text { to } \\
100.46 \%\end{array}$ & $\begin{array}{l}0.744- \\
1.507\end{array}$ & 530 & 0.22 \\
\hline Brento Tablet & $\begin{array}{l}\text { Chloroform- Ethyl } \\
\text { acetate- Toluene- glacial } \\
\text { acetic acid }(2: 5: 5: 1) \\
\mathrm{v} / \mathrm{v} / \mathrm{v} / \mathrm{v})\end{array}$ & 0.478 & 0.037 & 0.112 & $\begin{array}{l}99.14 \pm \\
0.465\end{array}$ & 0.435 & 223 & $\begin{array}{l}0.27 \pm \\
0.02\end{array}$ \\
\hline
\end{tabular}


Different plant parts of Withania somnifera have been used for the quantification and validation of Withanolide A, 1,2 Deoxy-withastranolide, Withanolide
B, 12-Deoxywithastramonolide are mentioned in table 8.

Table-8: Validation \& quantification of withanolides (other than withaferin-a) by HPTLC using different plant parts of $W$. somnifera (Devkar et al., 2012, Srivastava et al., 2008)

\begin{tabular}{|c|c|c|c|c|c|c|c|c|c|}
\hline Compound & $\begin{array}{l}\text { Plant } \\
\text { part } \\
\text { used }\end{array}$ & Mobile phase- & $\begin{array}{l}\text { Amount } \\
\text { present }\end{array}$ & $\begin{array}{l}\text { LOD } \\
\text { (ng) }\end{array}$ & $\begin{array}{l}\text { LOQ } \\
\text { (ng) }\end{array}$ & $\begin{array}{l}\text { Average } \\
\text { recovery } \\
(\%) \\
\end{array}$ & $\begin{array}{l}\text { Precision } \\
\text { (\%) RSD- }\end{array}$ & $\begin{array}{l}\text { absorption } \\
\text { reflection } \\
\text { mode at }\end{array}$ & $\begin{array}{l}\mathbf{R}_{\mathrm{f}} \\
\text { values }\end{array}$ \\
\hline Withanolide A & \multirow[t]{3}{*}{ Root } & \multirow{3}{*}{$\begin{array}{l}\text { Diethyl ether: } \\
\text { acetone: methanol: } \\
\text { toluene: } \\
\text { dichloromethane } \\
(1: 1: 3: 7.5: 7.5 \\
\text { v/v/v/v/v }\end{array}$} & 2.78 & 60 & 250 & 98 & 1.75 & 235 & 0.68 \\
\hline $\begin{array}{l}\text { 1,2 Deoxy- } \\
\text { withastranolide }\end{array}$ & & & 1.12 & 80 & 300 & 99.5 & 1.14 & 235 & 0.61 \\
\hline Withanolide B & & & 2.19 & 80 & 300 & 99 & 1.60 & 235 & 0.79 \\
\hline $\begin{array}{l}\text { 12- } \\
\text { Deoxywithastra } \\
\text { monolide }\end{array}$ & $\begin{array}{l}\text { Plant } \\
\text { tissues }\end{array}$ & $\begin{array}{l}\text { Acetone: methanol- } \\
\text { dichloromethane } \\
\text { diethyl ether } 1: 1: 15: 1\end{array}$ & 0.094 & 13.66 & 45.55 & $\begin{array}{l}98.00- \\
101.25\end{array}$ & $0.18-0.74$ & 230 & $\begin{array}{l}0.72 \pm \\
0.03\end{array}$ \\
\hline withanolide-A & $\begin{array}{l}\text { Plant } \\
\text { tissues }\end{array}$ & $\begin{array}{l}\text { Acetone: methanol- } \\
\text { dichloromethane } \\
\text { diethyl ether } 1: 1: 15: 1\end{array}$ & 23.13 & 0.109 & 72.76 & $\begin{array}{l}95.83- \\
98.95\end{array}$ & $0.18-0.85$ & 675 & $\begin{array}{l}0.86 \pm \\
0.03\end{array}$ \\
\hline
\end{tabular}

Marketed formulations like vati, capsule, churna and ashwagandharistha has been quantified and validated by HPTLC for the determination of $\beta$ sitosterol D glucoside, gallic acid and Rutin are mentioned in table 9. All developed HPTLC methods were validated in terms of LOD, LOQ, precision, accuracy and specificity. For the quality determination and standardisation of polyherbal preparations, it is critical to combine modern scientific knowledge with sensitive analytical techniques.

Table-9: Validation \& quantification of withanolides (other than withaferin-a) by HPTLC using marketed formulations of W. somnifera (Tatke et al., 2010, Bhondave et al., 2014, Tiwari et al., 2012)

\begin{tabular}{|c|c|c|c|c|c|c|c|c|c|}
\hline Compound & $\begin{array}{l}\text { Marketed } \\
\text { Formulations }\end{array}$ & Mobile phase- & $\begin{array}{l}\text { Amount } \\
\text { present }\end{array}$ & $\begin{array}{l}\text { LO } \\
\text { D } \\
\text { (ng) }\end{array}$ & $\begin{array}{l}\text { LOQ } \\
\text { (ng) }\end{array}$ & $\begin{array}{l}\text { Average } \\
\text { recovery } \\
(\%)\end{array}$ & $\begin{array}{l}\text { Precision } \\
(\%) \\
\text { RSD- }\end{array}$ & $\begin{array}{l}\text { absorption } \\
\text { reflection } \\
\text { mode at }\end{array}$ & $\begin{array}{l}\mathbf{R}_{\mathbf{f}} \\
\text { values }\end{array}$ \\
\hline $\begin{array}{l}\beta \text {-sitosterol D } \\
\text { glucoside }\end{array}$ & Capsule & $\begin{array}{l}\text { methanol: } \\
\text { chloroform } 2: 8 \mathrm{v} / \mathrm{v}\end{array}$ & 0.293 & 10 & 30 & $\begin{array}{l}97.13 \\
\pm 0.09\end{array}$ & 0.007 & 207nm) & 0.21 \\
\hline $\begin{array}{l}\beta \text {-sitosterol D } \\
\text { glucoside }\end{array}$ & Aristha & $\begin{array}{l}\text { methanol: } \\
\text { chloroform } 2: 8 \mathrm{v} / \mathrm{v}\end{array}$ & 0.250 & 10 & 30 & $99.00 \pm 0.09$ & 0.069 & 207nm) & 0.21 \\
\hline $\begin{array}{l}\beta \text {-sitosterol D } \\
\text { glucoside }\end{array}$ & Vati & $\begin{array}{l}\text { methanol: } \\
\text { chloroform } 2: 8 \mathrm{v} / \mathrm{v}\end{array}$ & 0.278 & 10 & 30 & $\begin{array}{l}98.67 \\
\pm 0.10\end{array}$ & 0.067 & 207nm) & 0.21 \\
\hline $\begin{array}{l}\beta \text {-sitosterol D } \\
\text { glucoside }\end{array}$ & Churna & $\begin{array}{l}\text { methanol: } \\
\text { chloroform } 2: 8 \mathrm{v} / \mathrm{v}\end{array}$ & 0.234 & 10 & 30 & $98.06 \pm 0.22$ & 0.057 & $207 \mathrm{~nm}$ & 0.21 \\
\hline Gallic acid & $\begin{array}{l}\text { Ashvagandhar } \\
\text { ishta }\end{array}$ & $\begin{array}{l}\text { toulene:: formic } \\
\text { acid: methanol: } \\
\text { ethyl acetate } \\
(6: 0.4: 0.2: 3)\end{array}$ & 0.064 & $\begin{array}{l}17.7 \\
444\end{array}$ & $\begin{array}{l}53.77 \\
11\end{array}$ & $\begin{array}{l}98.77- \\
101.96\end{array}$ & $\begin{array}{l}0.64- \\
0.78\end{array}$ & 474 & 0.26 \\
\hline Rutin & & $\begin{array}{l}\text { toulene:: formic } \\
\text { acid: methanol: } \\
\text { ethyl acetate } \\
(6: 0.4: 0.2: 3)\end{array}$ & 0.00464 & 120 & 400 & 99.86 & 0.76 & 280 & 0.59 \\
\hline
\end{tabular}

\section{CONCLUSION}

Quality control is a very important aspect for the safety and efficacy of herbal formulations. And to maintain the quality of marketed formulations validation plays an important role. For this purpose validation parameters were studied and discussed. Different validation methods were used to standardize different kind of formulations containing Ashwagandha by HPTLC.
HPTLC methods can be used to determine batch to batch variations and routine analysis by he rbal manufacturers of its formulations. Due to potential behaviour of $W$. somnifera in many diseases, it has been used from so many years but more clinical trials are still required for better therapeutic efficacy. So there is a need for more research work to maintain the quality of herbal formulations for quantification as well as qualification of withaferin-A, withanolide-A, B and D in crude drug as well as in marketed formulations. 


\section{REFERENCES}

1. Sharma, A., Shanker, C., Tyagi, L. K., Singh, M., \& Rao, C. V. (2008). Herbal medicine for market potential in India: an overview. Acad $J$ Plant Sci, 1(2), 26-36.

2. Balekundri, A., \& Mannur, V. (2020). Quality control of the traditional herbs and herbal products: a review. Future Journal of Pharmaceutical Sciences, 6(1), 1-9.

3. Gupta, G. L., \& Rana, A. C. (2007). PHCOG MAG.: Plant review Withania somnifera (Ashwagandha): A review. Pharmacognosy Reviews, 1(1), 129-136.

4. Singh, N., Bhalla, M., de Jager, P., \& Gilca, M. (2011). An overview on ashwagandha: a Rasayana (rejuvenator) of Ayurveda. African Journal of Traditional, Complementary and Alternative Medicines, 8(5S), 208-213.

5. Meher, S. K., Das, B., Panda, P., Bhuyan, G. C., \& Rao, M. M. (2016). Uses of Withania somnifera (Linn) Dunal (Ashwagandha) in Ayurveda and its pharmacological evidences. Research Journal of Pharmacology and Pharmacodynamics, 8(1), 23-29.

6. Saleem, S., Muhammad, G., Hussain, M. A., Altaf, M., \& Bukhari, S. N. A. (2020). Withania somnifera L.: Insights into the phytochemical profile, therapeutic potential, clinical trials, and future prospective. Iranian Journal of Basic Medical Sciences, 23(12), 1501-1526.

7. Krutika, J., Tavhare, S., Panara, K., Kumar, P., \& Karra, N. (2016). Studies of ashwagandha (Withania somnifera dunal). Int J Pharm Biol Arch, 7(1), 1-11.

8. Modi, V., Bhatkar, S., Prajapati, P., \& Basuri, T. A. (2016). A review on various applications of high performance thin layer chromatography (HPTLC) in pharmaceuticals. International Journal of Innovative Pharmaceutical Sciences and Research,4(3), 386387.

9. Sonia, K., Beddi, B., Lakshmi, K.S. (2017). HPTLC Method Development and Validation: An Overview. Journal of Pharmaceutical Sciences and Research, 9(5), 652-657.

10. Ahir, K. B., Singh, K. D., Yadav, S. P., Patel, H. S., \& Poyahari, C. B. (2014). Overview of validation and basic concepts of process validation. Sch. Acad. J. Pharm, 3(2), 178.

11. Jyothi, D. I., Khanam, S. A., \& Sultana, R. O., (2010). Optimization of microwave assisted extraction of withanolides from roots of ashwagandha and its comparison with conventional extraction method. Int. J. Pharm Sci, 2(4), 46-50.

12. Jain, H., Parial, S. D., Jarald, E., Daud, A. S., \& Ahmad, S. (2010). Extraction of Ashwagandha by conventional extraction methods and evaluation of its anti-stress activity. International Journal of Green Pharmacy (IJGP), 4(3).

13. Dhanani, T., Shah, S., Gajbhiye, N. A., \& Kumar, S. (2017). Effect of extraction methods on yield, phytochemical constituents and antioxidant activity of Withania somnifera. Arabian Journal of Chemistry, 10, S1193-S1199.

14. Kherde, S. D., Parmar, K. M., Tawar, M. G., Prasad, S. K., \& Itankar, P. R. (2020). Study on impact of different climatic zones on physicochemical and phytochemical profile of Withaniasomnifera (L.) Dunal.

15. Kumar, M. S., Kumar, D. V., Ramachandran, A., \& Shajahan, A. (2010). Anatomical studies in Withania somnifera L. Dunal)—an important medicinal plant. Adv Biotechnol, 10(3), 44-46.

16. Srivastava, P., Tiwari, N., Yadav, A. K., Kumar, V., Shanker, K., Verma, R. K., \& Khanuja, S. P. (2008). Simultaneous quantification of withanolides in Withania somnifera by a validated highperformance thin-layer chromatographic method. Journal of AOAC International,91(5), 1154-1161.

17. Sharma, V., Gupta, A. P., Bhandari, P., Gupta, R. C., \& Singh, B. (2007). A validated and densitometric HPTLC method for the quantification of Withaferin-A and Withanolide-A in different plant parts of two morphotypes of Withania somnifera. Chromatographia, 66(9), 801-804.

18. Devkar, S., Badhe, Y., Jagtap, S., \& Hegde, M. (2012). Quantification of major bioactive withanolides in Withania somnifera (Ashwagandha) roots by HPTLC for rapid validation of Ayurvedic products. JPC-Journal of Planar ChromatographyModern TLC, 25(4), 290-294.

19. Sangwan, R. S., Chaurasiya, N. D., Misra, L. N., Lal, P., Uniyal, G. C., Sharma, R., \& Tuli, R. (2004). Phytochemical variability in commercial herbal products and preparations of Withania somnifera (Ashwagandha). Current Science, 461465.

20. Mirjalili, M. H., Fakhr-Tabatabaei, S. M., Alizadeh, H., Ghassempour, A., \& Mirzajani, F. (2009). Genetic and withaferin a analysis of Iranian natural populations of Withania somnifera and W. coagulans by RAPD and HPTLC. Natural product communications, 4(3), 337-346.

21. Nayak, P., Upadhyaya, S., \& Upadhyaya, A. (2009). HPTLC method for analysis of withaferin-A in Ashwagandha (Withania somnifera). JPC-Journal of Planar Chromatography-Modern TLC, 22(3), 197-200.

22. Tomar, V., Beuerle, T., \& Sircar, D. (2019). A validated HPTLC method for the simultaneous quantifications of three phenolic acids and three withanolides from Withania somnifera plants and its herbal products. Journal of Chromatography $B, 1124,154-160$.

23. Tatke, P., Jigre, S., Gabhe, SY. (2010). Marker based standardization of commercial formulations and extracts containing beta-sitosterol D glucoside using HPTLC. International Journal of Research of Ayurveda \& Pharmacy, 1, 616-623.

24. Bhondave, P. D., Potawale, S. E., Gabhe, S. Y., \& Harsulkar, A. M. (2014). A rapid and simple high 
performance thin layer chromatographic method for simultaneous analysis of [beta]-sitosterol-Dglucoside, gallic acid, withaferin A and withanolide A in Ashvagandharishta. Journal of Applied Pharmaceutical Science, 4(7), 82-87

25. Mistry, N. N., Shah, P., Patel, K., \& Hingorani, L. (2015). Simultaneous estimation of stigmasterol and Withaferin A in union total herbal formulation using validated HPTLC method. Journal of Applied Pharmaceutical Science, 5(08), 159-166.

26. Trivedi Manisha N, Santani DD. Pelagia Research Library.
27. Tiwari, P., Patel, R.K. (2012). Quantification of quercetin and rutin in Ashwagandharistha by validated HPTLC Densitometry. Asian J. Research Chem. 5,441-445.

28. Patil, S. T., Ahirrao, R. A., \& Pawar, S. P. (2017). A short review on method validation. Journal of Pharmaceutical and BioSciences, 5(4), 30-37.

29. Rashmin, P., Mrunali, P., \& BHARAT, P. (2012). HPTLC method development and validation: strategy to minimize methodological failures. Journal of food and drug analysis, 20(4), 794-804. 